\title{
Comparative analysis of disperse characteristics of single-type flat spray cone nozzles
}

\author{
E.G. Aristov*, N.N. Krakhovetsky, V.G. Selivanov, and N.P. Mishurov
}

Russian Research Institute of Information and Feasibility Study on Engineering Support of Agribusiness, the Federal State Budgetary Scientific Institution (Rosinformagrotekh FSBSI), 60, Lesnaya Str., Pravdinsky Township, 141261 Moscow Region, Russian Federation

\begin{abstract}
Research and development work related to the study and use of water spray must certainly be based on the most accurate determination of the dispersion composition of spray droplets. It is just this parameter that forms the basis for all further calculations of the effect of water spray on the process cycle components. The introduction of the method for restoring the original sizes of spray droplets and the most accurate measurement of their disperse characteristics will allow coming close to solving the problem of optimizing the operation of spray technology, as well as will make it possible to predict and possibly regulate the dispersed characteristics of droplet flows. Therefore, it is the foundation for the development of breakthrough and environmentally sustainable resource-saving technologies in the agricultural sector. We have developed a method for restoring the original size of the droplet spectrum (Patent No. 2709402 titled "A method for determining the size of droplets"). Based on this method, a test bench was developed and manufactured to assess the quality of spraying of working fluids. An aerosol chamber design was developed and the chamber was assembled, in which an aerosol sample was taken under conditions of equal probability of deposition and preservation of droplets of all sizes. Software for high-speed measurement of the dispersion characteristics of the spectrum of spray droplets has been developed. In general, the studies performed have demonstrated the appropriate professional capabilities of the experimental facilities when testing sprayers. This gives us an opportunity to come up with a proposal to create centers for testing sprayers for their compliance with the declared performances based on the experimental facilities.
\end{abstract}

\section{Introduction}

Over the past 10-15 years, up-to-date biologically super-active herbicides having low (5 to $30 \mathrm{~g} / \mathrm{ha}$ ) consumption rates are being applied into agricultural practice more and more. Spraying machines and agricultural technologies existing today in the world are generally unsuitable for their application. Therefore, the rate of entry into nature of all poisons involved

\footnotetext{
${ }^{*}$ Corresponding author: $\underline{\text { mgp1947@mail.ru }}$
} 
in processes of chemical protective measures more and more outstrips the rate of detoxification of these poisons by nature.

Creation of spray equipment designs having controlled disperse characteristics is the basis for the development of breakthrough and environmentally friendly resource-saving technologies in the agricultural sector. They open up a real opportunity to reduce the ecotoxicant load on agrocenoses and the human environment and significantly reduce the specific consumption of agrochemicals.

Research objective: Measurement of the numerical values of the dispersion characteristics of the spray cone, its geometry and heterogeneity over the irrigation field is an absolutely mandatory step, without which it is impossible to assess the efficiency of the spraying process, select the optimal parameters for chemical plant protection measures, and ensure the environmental safety of the process.

\section{Materials and research methods}

Designing a new spray technique or optimizing an existing one is possible with reliable technology for quantitative spray testing. We have developed a method for measuring droplet sizes that returns the original pattern to the spray spectrum transformed as a result of interaction with the external environment. It is obviously that to obtain a reliable quantitative result when determining the numerical characteristics of drops, we can apply the method we have created for restoring their original sizes $[1,2,3]$.

The paper presents processes, hardware and software for measuring the dispersion characteristics of the spray cone at different stages of its development.

\section{Research results}

The experiments were carried out in a spray chamber (on a measuring stand.) Three uniform (ST) nozzles were selected: \#1 nozzle having a jet size of $5.1 \times 2 \mathrm{~mm}$, \#2 nozzle having a jet size of $4.5 \times 2.2 \mathrm{~mm}$., and \#3 nozzle having a jet size of $3.5 \times 2.4 \mathrm{~mm}$ (Fig. 1.) Differences in the size and shape of the nozzle jets should obviously affect the spectrum of the droplets formed $[4,5]$.

Experimental conditions were identical for all nozzles. 

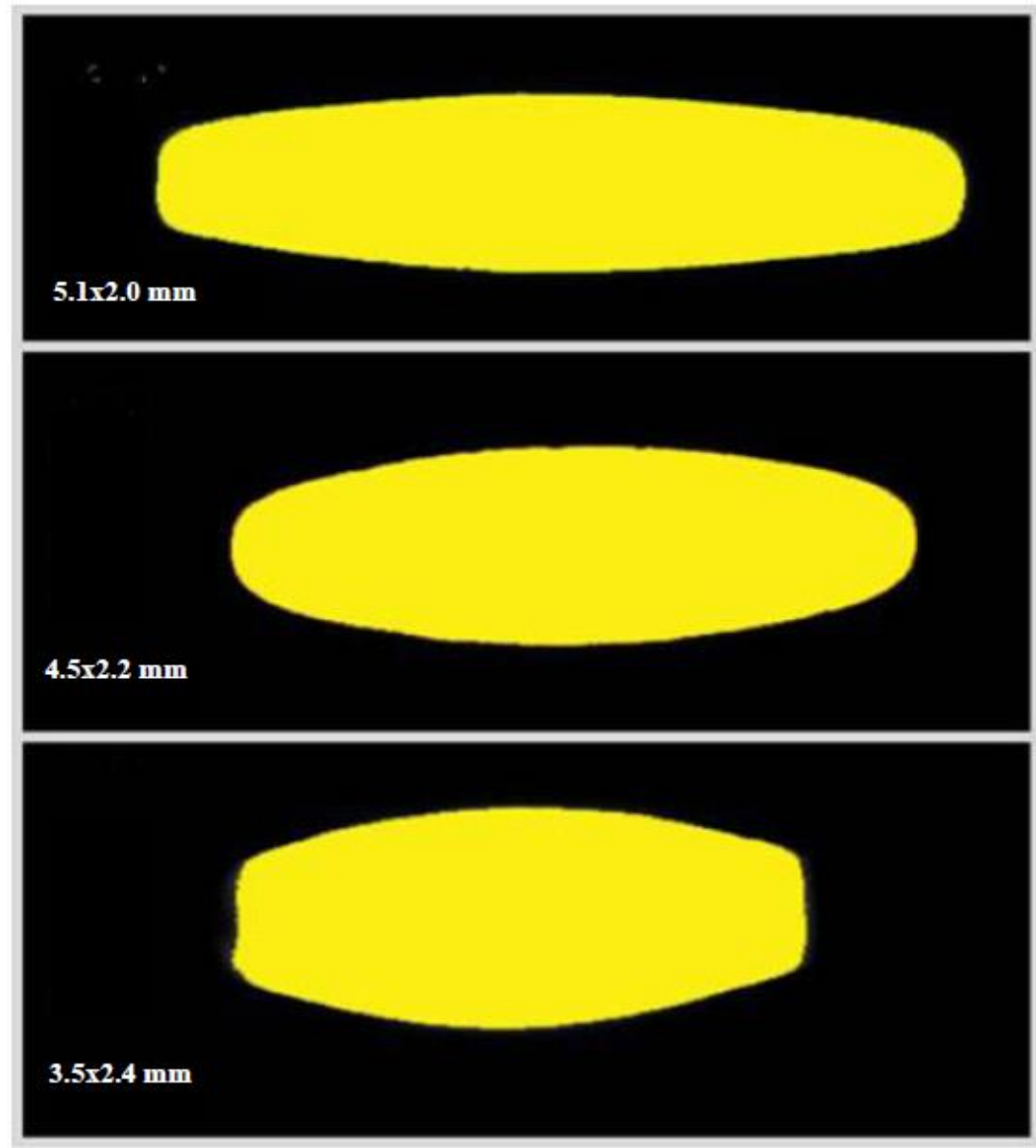

Fig. 1. Nozzle jet shape and size

The nozzle was mounted on a rod under the ceiling of the chamber. The nozzle jet was positioned parallel to the chamber wall. Samplers were installed under the nozzle at a distance of $1 \mathrm{~m}$ along a line oriented parallel to the nozzle jet. A total of 4 samplers were installed, thus, information on the dispersed composition of droplets came from four zones of the irrigation field. Zero zone was directly under the nozzle, zone \#1 was at a distance of $25 \mathrm{~cm}$ from the zero zone and further zones $\# 2$ and $\# 3$ were placed at a $25 \mathrm{~cm}$ increment from each other.

Slides coated with a water-repellent layer were placed on the samplers.

The $5 \% \mathrm{NaCl}$ solution was fed into the nozzle at a pressure of 4 bars with the shutter closed. Only after the nozzle had achieved the set operating conditions, the shutter was moved back and the droplets entered the chamber [6]. The exposure time was kept equal to approximately $1 \mathrm{~s}$. Then the shutter blocked the spray access to the chambers and the solution supply was turned off. The slides were kept in the chamber for about half an hour until the droplets were completely deposited and then transferred to the recovery chamber. It should be noted that during the holding time, complete evaporation of the droplet was not observed, which was obviously associated with a sufficiently high humidity in the chamber and the presence of a hygroscopic salt in the solution. But even in the case of complete evaporation 
of the liquid, the $\mathrm{NaCl}$ crystal, a non-evaporating information core, would retain all the data on the initial parameters of the drop.

The exposed slides were transferred to a recovery chamber and placed on a microscope stage. The digital camera of the microscope transmitted in real time the image of the droplets to the computer monitor. To begin with, a control snapshot of the observed drops was taken. The object stage did not move and, therefore, it was possible, by comparing the current image of drops with the control one, to record the completion of the process of restoring the size of drops.

Then the digital registration of the image of the drops was performed. The area of each individual frame was about $6 \mathrm{~mm}^{2}$. The number of frames in each zone was chosen so that the total number of drops was at least 600 pieces. After collecting the required amount of the snapshot, the image was graphically processed using dedicated software we had developed [2].

Comparison of photographs of droplets in different zones of the irrigation field showed a rather high degree of heterogeneity both in terms of disperse characteristics and in terms of the density of droplet concentration.

Figure 2 shows photographs of drops deposited on glass slides in different zones of the irrigation field.

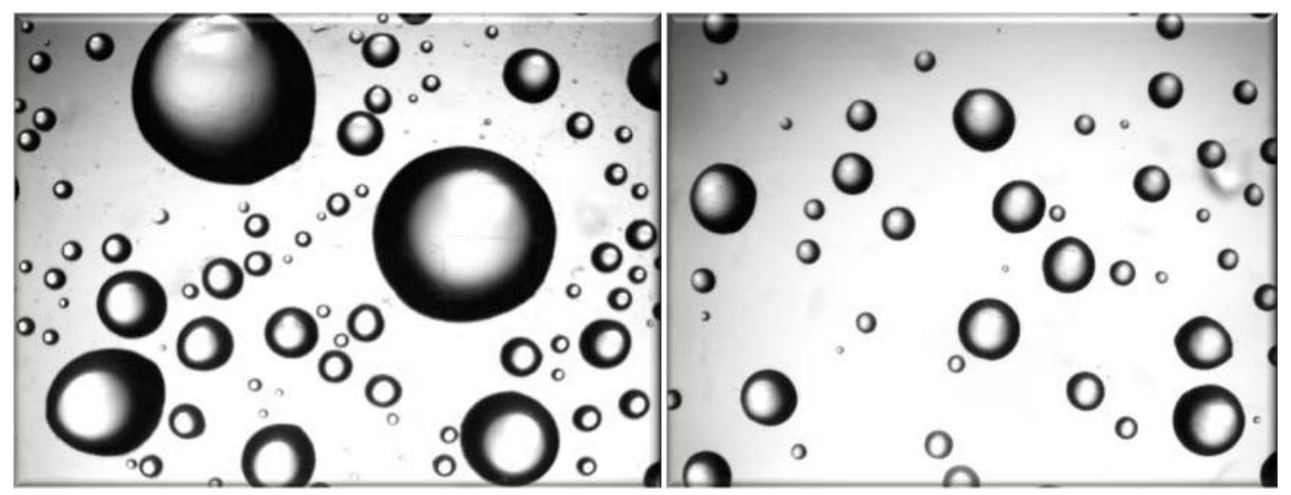

Fig. 2a. (zone 0)

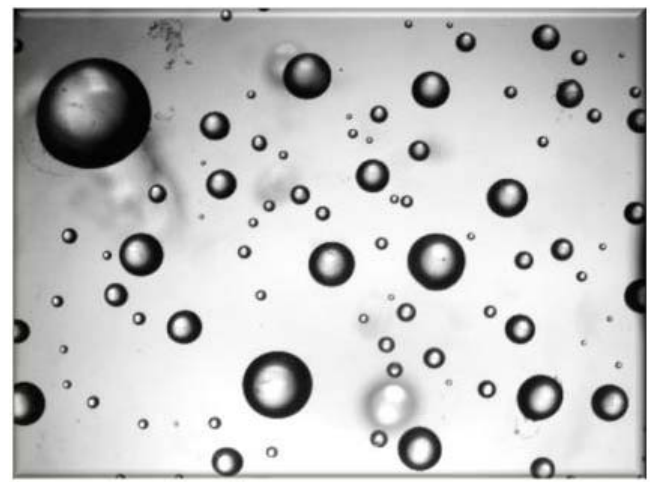

Fig. 2c. (zone 2)
Fig. 2b. (zone 1)

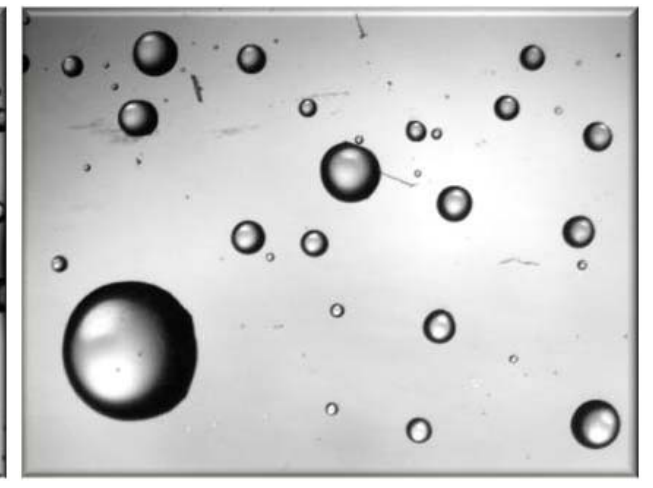

Fig. 2d. (zone 3)

Visual analysis of photographs indicates, first of all, a large number of small droplets in the spray spectrum. This is especially the case in the central zone, that is to say, in zone 0 . At the same time, there are gigantic droplets, which indicates a high degree of polydispersity. In 
zone 1, the number of small droplets and, consequently, the degree of polydispersity is much lower. Zones 2 and 3 are characterized by a lower concentration of droplets. The degree of polydispersity is average.

In order to evaluate in detail the results obtained, we will conduct a quantitative analysis of the dispersed parameters of the spray droplets of the nozzles investigated.

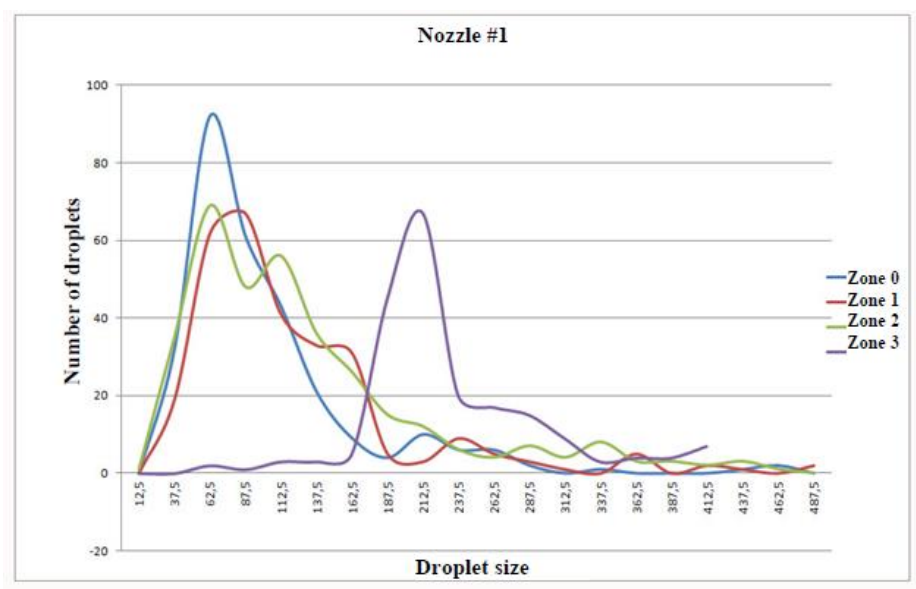

Fig. 3. Diagram of the distribution by the size of spray droplets of nozzle \#1 in different zones.

As follows from the curves shown (Fig. 3), the modal sizes in the first three zones differ little from each other. The value of the modal diameter ranges from 60 to 80 microns. In zone 3 , there is a sharp increase in the size of the modal diameter up to $220 \mu \mathrm{m}$. A similar case is observed with average linear dimensions too. The values of the average diameters remain in the range of 130 to 150 microns. Within the limits of error, they can be considered equal. However, there is a sharp jump up to 230 microns in zone 3.

The situation is different with the average volumetric diameter. The average volumetric diameter just is maximum one in zone 0 . This is despite the fact that the modal and average linear diameters in this zone are the smallest. This indicates a high degree of polydispersity of the droplet spectrum in this zone. The minimum value of the degree of polydispersity is observed in zone 3.

Modal, average linear and average volumetric diameters differ little from each other. The droplet spectrum of this particular zone is most preferable when performing the chemical plant protection.

Table 1 shows the numerical values of the modal, linear and volumetric diameters in different zones of the irrigation field.

Table 1. Diameter numerical values. (Nozzle \#1)

\begin{tabular}{|c|c|c|c|}
\hline & Mode [micron] & $\begin{array}{c}\text { Average linear } \\
\text { diameter [micron] }\end{array}$ & $\begin{array}{c}\text { Average } \\
\text { volumetric } \\
\text { diameter [micron] }\end{array}$ \\
\hline Zone 0 & 60 & 128 & 318 \\
\hline Zone 1 & 80 & 147 & 282 \\
\hline Zone 2 & 65 & 131 & 191 \\
\hline Zone 3 & 220 & 233 & 251 \\
\hline
\end{tabular}

A similar pattern is observed in the spray spectrum of nozzle \#2 (Fig. 4). Similar to the operation of nozzle \#1, the distribution mode is shifted towards larger sizes in zone 3. 


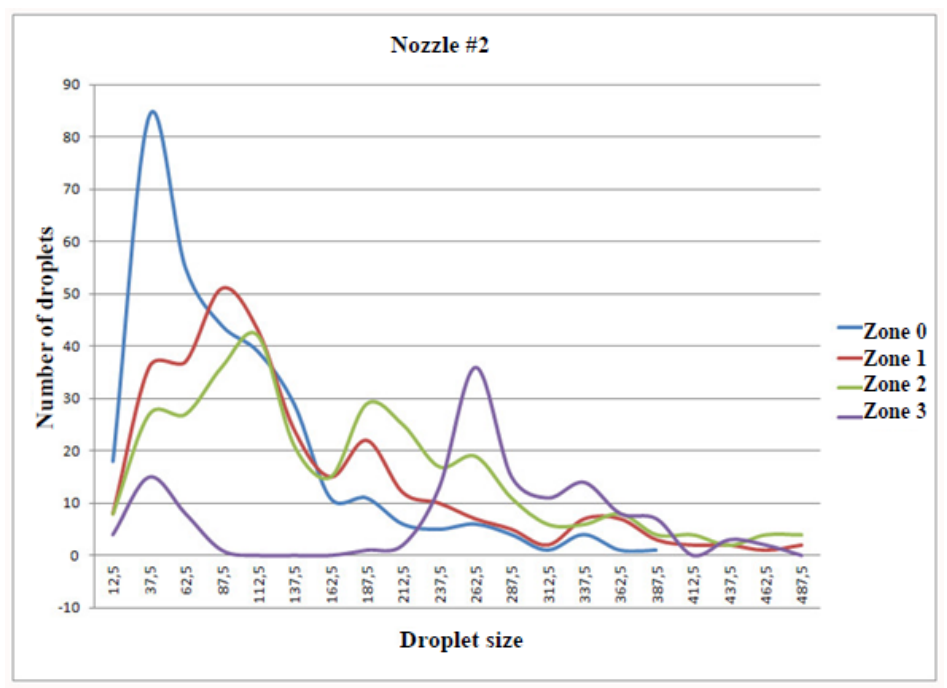

Fig. 4. Diagram of the distribution by the size of spray droplets of nozzle \#2 in different zones.

Table 2 shows the changes in the numerical values of diameters.

Table 2. Diameter numerical values. (Nozzle \#2)

\begin{tabular}{|c|c|c|c|}
\hline & Mode [micron] & $\begin{array}{c}\text { Average linear } \\
\text { diameter [micron] }\end{array}$ & $\begin{array}{c}\text { Average } \\
\text { volumetric } \\
\text { diameter [micron] }\end{array}$ \\
\hline Zone 0 & 40 & 97 & 148 \\
\hline Zone 1 & 90 & 145 & 224 \\
\hline Zone 2 & 115 & 183 & 266 \\
\hline Zone 3 & 260 & 247 & 288 \\
\hline
\end{tabular}

The distribution of droplet sizes in different zones has a different character when the working fluid is sprayed through the nozzle \#3.

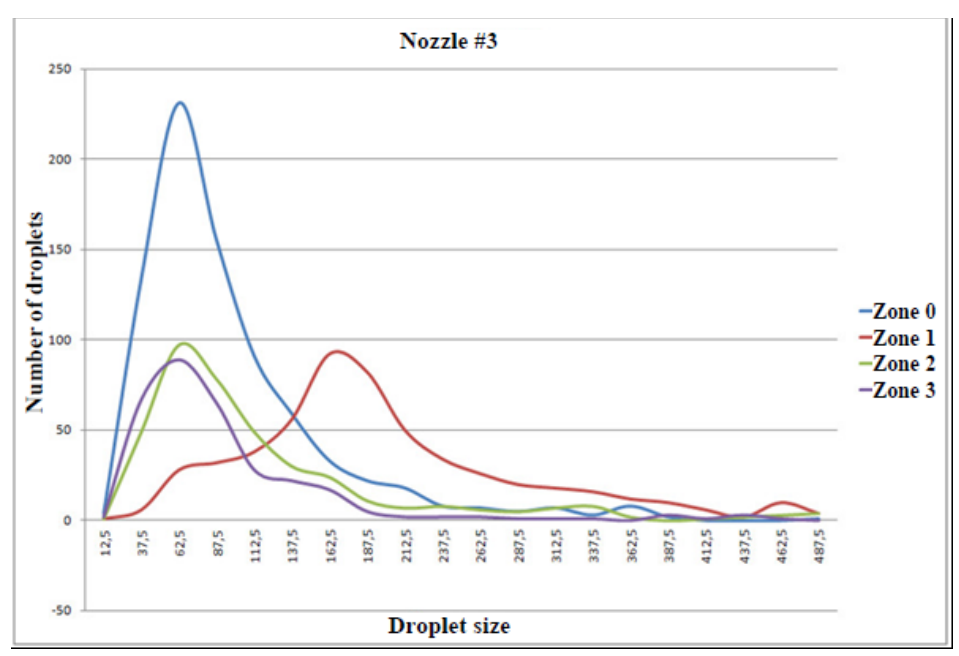

Fig. 5. Diagram of the distribution by the size of spray droplets of nozzle \#3 in different zones. 
As follows from a comparison of the diagrams shown in Fig. 3, Fig. 4 and Fig. 5, the shift of the distribution mode towards large sizes is observed not in zone 3, as it is observed for nozzles $\# 1$ and $\# 2$, but in zone 2 . Accordingly, in this zone the convergence of the dimensional parameters of the spray spectrum is also observed.

Table 3 shows the changes in the numerical values of the diameters shown in Fig. 5.

Table 3. Diameter numerical values. (Nozzle \#3)

\begin{tabular}{|c|c|c|c|}
\hline & Mode [micron] & $\begin{array}{c}\text { Average linear } \\
\text { diameter [micron] }\end{array}$ & $\begin{array}{c}\text { Average } \\
\text { volumetric } \\
\text { diameter [micron] }\end{array}$ \\
\hline Zone 0 & 60 & 122 & 334 \\
\hline Zone 1 & 160 & 200 & 244 \\
\hline Zone 2 & 60 & 126 & 211 \\
\hline Zone 3 & 60 & 100 & 170 \\
\hline
\end{tabular}

Let us compare the experimental curves of the size distribution of the droplet spectra of each of the nozzles by zones.

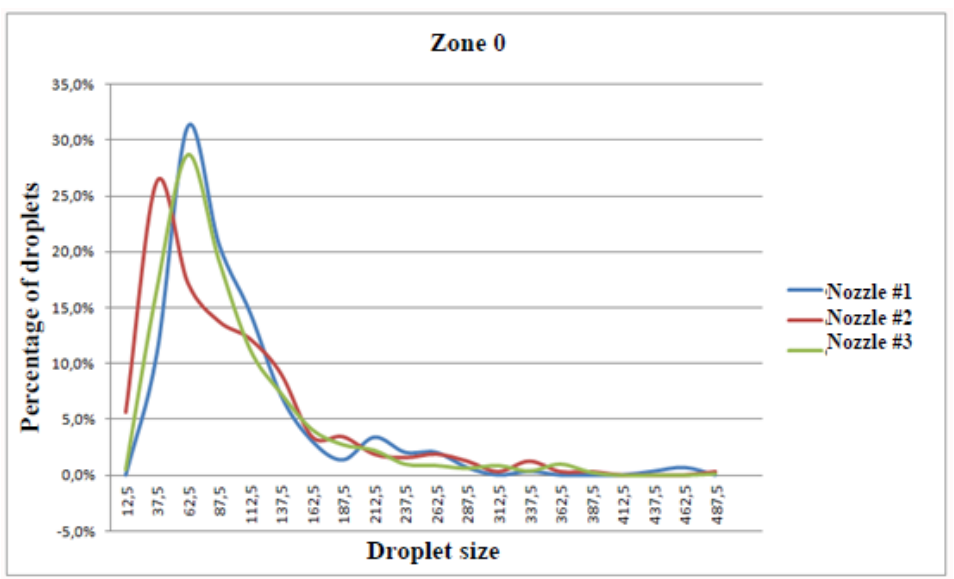

Fig. 6. Experimental droplet spectra size distribution curves in zone 0 .

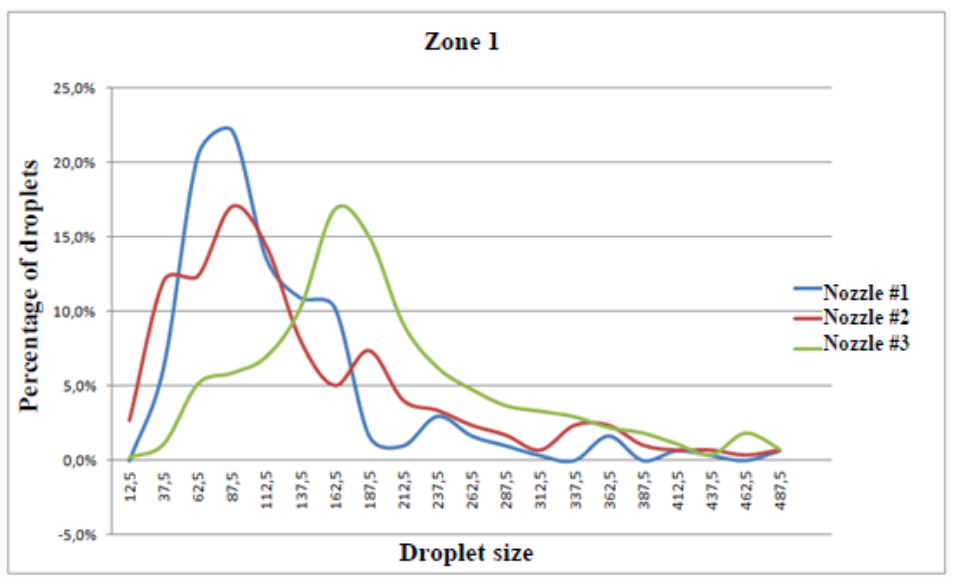

Fig. 7. Experimental droplet spectra size distribution curves in zone 1. 


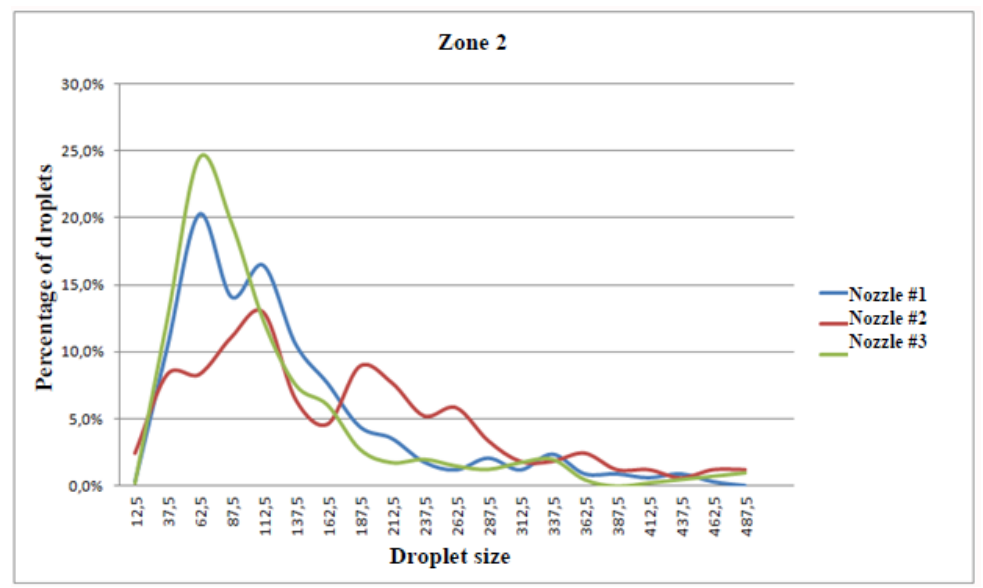

Fig. 8. Experimental droplet spectra size distribution curves in zone 2.

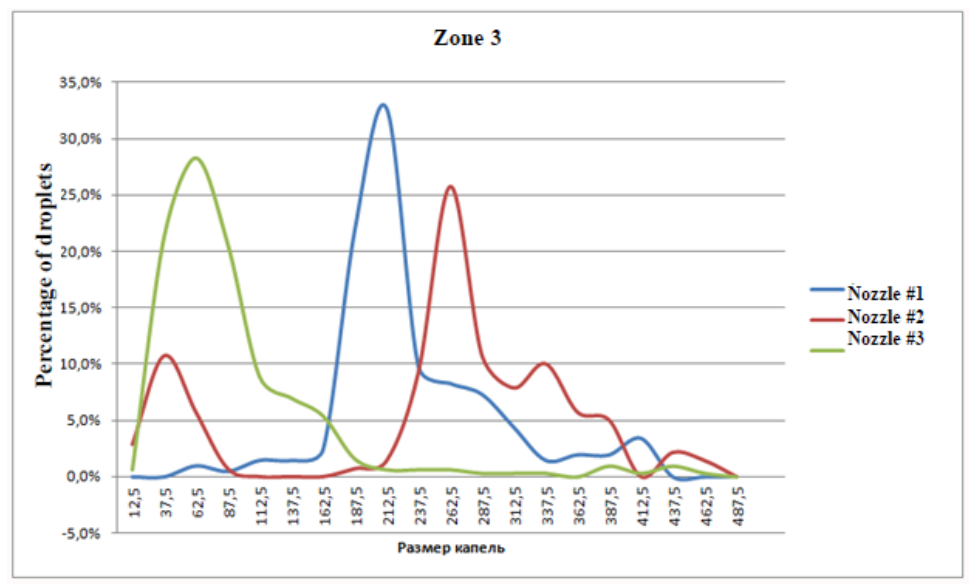

Fig. 9. Experimental droplet spectra size distribution curves in zone 3.

\section{Conclusions}

Analysis of the diagrams allows us to conclude that the nature, inhomogeneity and its distribution on the irrigation field for nozzles \#1 and \#2 have a similar orientation, while the inhomogeneity of the irrigation field of the nozzle \#3 has a different geometry.

With what it can be connected? It is possible that the geometry of the nozzle has an influence. Figure 1 shows macro photographs of the nozzle jets. The shapes and sizes of all three nozzles differ from each other. It can be assumed that this is the reason for the differences in their operation. Of course, all these findings are preliminary.

In general, the analysis of the results obtained allows us to conclude that there are a large number of small drops and a high degree of polydispersity of the spectrum of the drops formed by these nozzles. The observed heterogeneity of the quantitative characteristics of the dispersed fraction in the irrigation field indicates significant differences in these parameters in the zones studied.

The results obtained cannot be considered final. The experiments should be continued according to an extended program, the purpose of which is, in addition to determining the 
test characteristics of spray mechanisms, to reveal a possible relationship between the design features of the nozzles and the spectrum of dispersed droplets.

Based on the results of experiments, it can be stated that the method of restoring the original droplet size is an absolutely reliable and correct method and can be recommended for widespread use in test tests of spray technology.

\section{References}

1. I. Ya. Paremsky, V. F. Fedorenko, E. G. Aristov, V. G. Selivanov and N. N. Krakhovetsky, Process and technical means for quantitative assessment of dispersion characteristics of chemical plant protection sprayers. Int. Conf. on modern trends in the development of production processes and equipment (ICMTMTE 2020) (2020)

2. Research report, Research and development of technology with technical means of quality control of sprayers for chemical plant protection based on an innovative method of restoring the original sizes of spray droplets, (Rosinformagrotekh) (2019)

3. Method for determining droplet sizes, Invention patent \#2709402 (2019)

4. D.G. Pazhy, V.S. Galustov, Fundamentals of liquid spraying - Processes and devices of chemical and petrochemical technology (Moscow: Chemistry Publishers, 1984)

5. C. Gong, Y. Ma, R. Yang, X. Wang, Y. Liu, Scientia Agricultura Sinica, (2020)

6. Research report, Research and development of technology for express testing of spraying devices of machines for chemical plant protection using a spray chamber, (Rosinformagrotekh) (2020) 